\title{
Intermolecular Bond Lengths: Extrapolation to the Basis Set Limit on Uncorrected and BSSE-Corrected Potential Energy Hypersurfaces
}

\author{
BÉLA PAIZS, ${ }^{1}$ PEDRO SALVADOR, ${ }^{1, *}$ ATTILA G. CSÁSZÁR, ${ }^{2}$ \\ MIQUEL DURAN, ${ }^{3}$ SÁNDOR SUHAI ${ }^{1}$ \\ ${ }^{1}$ Department of Molecular Biophysics, German Cancer Research Center, Im Neuenheimer Feld 280, \\ D-69120 Heidelberg, Germany \\ ${ }^{2}$ Department of Theoretical Chemistry, Eötvös University, H-1518 Budapest 112, P.O. Box 32, Hungary \\ ${ }^{3}$ Institut de Química Computacional i Departament de Química, Universitat de Girona, 17071 Girona \\ (Catalonia), Spain
}

Received 11 January 2000; accepted 28 July 2000

\begin{abstract}
Geometry optimizations were carried out for the $(\mathrm{HF})_{2},\left(\mathrm{H}_{2} \mathrm{O}\right)_{2}$, and $\mathrm{HF}-\mathrm{H}_{2} \mathrm{O}$ intermolecular complexes using the MP2/aug-cc-pVXZ $\{X=2,3$, 4 , and 5$\}$ theoretical models on both the uncorrected and counterpoise (CP) corrected potential energy hypersurfaces (PES). Our results and the available literature data clearly show that extrapolation of intermolecular distances to the complete basis set (CBS) limit is satisfactory on PESs corrected for BSSE. On the other hand, one should avoid such extrapolations using data obtained from uncorrected PESs. Also, fixing intramolecular parameters at their experimental values could cause difficulties during the extrapolation. As the available literature data and our results clearly show, the MP2/aug-cc-pVXZ $\{X=2,3,4\}$ data series of intermolecular distances obtained from the $\mathrm{CP}$-corrected surfaces can be safely used for the purpose of CBS extrapolations. $\quad$ (C) 2000 John Wiley \& Sons, Inc. J Comput Chem 22: 196-207, 2001
\end{abstract}

Keywords: intermolecular bond length; ab initio; BSSE; CP-corrected; basis set limit

\footnotetext{
*Permanent address: Institut de Química Computacional i Departament de Química, Universitat de Girona, 17071 Girona (Catalonia), Spain de

Correspondence to: B. Paizs; e-mail: b.paizs@dkfz-heidelberg.

Contract/grant sponsor: CIRIT (to P. Salvador); contract/ grant number: 1998FI-00564

Contract/grant sponsors: Hungarian Scientific Research Fund and Hungarian Ministry of Education (to A. G. Császár); contract/grant numbers: OTKA T024044 and FKFP 0117/1997
} 


\section{Introduction}

D uring the last few years significant progress has been made in the $a b$ initio computation of intermolecular interactions. The rapid development in quantum chemical procedures (including methods, basis sets, and computer codes) and the expansion of computational resources changed the prospects of theoreticians working in the field of noncovalent chemistry. From the relevant developments we mention only those of importance for the present study. First, Dunning and coworkers have developed a family of correlation consistent [(aug)cc-p $(\mathrm{C}) \mathrm{VXZ}, X=2(\mathrm{D}), 3(\mathrm{~T}), 4(\mathrm{Q}), \ldots]$ basis sets. ${ }^{1-3}$ These basis sets systematically extend the atomic radial and angular spaces as a function of the cardinal number $X$. Therefore, results obtained with them seem to provide an excellent opportunity to extrapolate energies as well as properties to the complete basis set (CBS) limit. In most systems studied, extrapolation of the total energy and many properties has been achieved with simple functional forms. The exponential form

$$
A_{\mathrm{X}}=A_{\mathrm{CBS}}+a e^{-b \mathrm{X}},
$$

and polynomials of the form

$$
A_{X}=A_{\mathrm{CBS}}+\sum_{k=3}^{k_{\max }} \alpha_{k}\left(X+\beta_{k}\right)^{-k},
$$

where $k_{\max }$ is small, or similar polynomial forms with noninteger exponents have been extensively employed to estimate the CBS limit. ${ }^{4-15}$ The form of eq. (1), for example, suggests that properties obtained with three basis sets (e.g., $X=\mathrm{D}, \mathrm{T}$, and $Q$ ) gives an opportunity to extrapolate to the CBS limit of the investigated property. Second, much experience ${ }^{4}$ has been accumulated on how the correlation-consistent basis sets can be employed in calculations on various properties. For example, it is clear that for calculations of intermolecular complexes ${ }^{5,13-15}$ one should apply the augmented version (aug-cc-pVXZ) of the cc-pVXZ sets containing diffuse functions, which are essential for the description of long-range interactions. Third, due to the rapid progress of both the available computer codes and hardware, it became practical to carry out $a b$ initio computations at correlated levels [Møller-Plesset (MPn), coupled cluster (CC)] employing basis sets of aug-cc-pVQZ quality or better for small intermolecular complexes.

One of the principal difficulties during computation of intermolecular properties is due to the basis set superposition error (BSSE). Briefly, because one cannot use complete basis sets in practical computations, the description of "internal" monomer properties depends on the quality and location of the basis functions of the partner molecule(s). Due to BSSE, the calculated interaction energies become too large, and the predicted potential energy hypersurfaces are distorted.

The conventional way to correct for BSSE $a$ posteriori is based on the Boys-Bernardi ${ }^{16,17}$ [counterpoise $(\mathrm{CP})]$ scheme. On the other hand, the Chemical Hamiltonian Approach ${ }^{18,19}$ (CHA) introduced by Mayer eliminates the nonphysical terms of the Hamiltonian that are due to BSSE a priori, and therefore, represents an ultimate solution to the problem of BSSE. The CHA is available up to the MP2 level of theory; ${ }^{20}$ however, the actual implementation is not suitable for calculations as large as those presented here. For an excellent recent review on CHA, see ref. 21.

Using the $\mathrm{CP}$ scheme one has to recalculate the monomers in the basis of the whole supermolecule for every geometrical arrangement. For example, in the case of two interacting monomers $A$ and $B$, the uncorrected interaction energy $(\Delta E)$ can be calculated as

$$
\Delta E=E_{A B}(A B)-E_{A}(A)-E_{B}(B),
$$

where $E_{A B}(A B)$ is the total energy of the complex, and $E_{A}(A)$ and $E_{B}(B)$ are the total energies of the monomers. [In the following, we will use subscripts to denote the molecular species in the energy expressions, while the letters in parentheses refer to the (composite) basis used in the calculation. For example, $E_{A}(A)$ is the energy of monomer $A$ calculated by using its own basis set.] The CP-corrected interaction energy can be defined as

$$
\Delta E^{\mathrm{CP}}=E_{A B}(A B)-E_{A}(A B)-E_{B}(A B) .
$$

Using eqs. (3) and (4), one can define the BSSE content of the interaction energy as

$$
\begin{aligned}
\delta^{\mathrm{BSSE}}=\Delta E-\Delta E^{\mathrm{CP}}=E_{A}(A B) & -E_{A}(A) \\
& +E_{B}(A B)-E_{B}(B) .
\end{aligned}
$$

Using eq. (5), one can define the CP-corrected potential energy surface (PES) of a dimer as

$$
\begin{array}{r}
E^{\mathrm{CP}}=E_{A B}(A B)-\delta^{\mathrm{BSSE}}=E_{A B}(A B)+E_{A}(A)-E_{A}(A B) \\
+E_{B}(B)-E_{B}(A B) .
\end{array}
$$

According to eq. (6), one has to calculate five different total energies ${ }^{22}$ at every geometrical arrangement of the system to determine a CP-corrected PES. Of course, eq. (6) can be generalized to the case of 
an arbitrary number of subsystems, but the number of energy calculations necessary to determine the PES increases with the number of monomers enormously. ${ }^{23}$

There has been a continuous debate on the $\mathrm{CP}$ method in the literature. One of the main arguments against the $\mathrm{CP}$ scheme is that the composite basis of the complex $A B$ is not available for monomer $A$, as the Pauli exclusion principle precludes the use of the occupied orbitals of monomer $B .^{24}$ In practice, this means that the $\mathrm{CP}$ scheme overcompensates the BSSE. This effect was studied on a small analytical model by Mayer and Túri. ${ }^{25}$ They found that the $\mathrm{CP}$ scheme takes the leading BSSE terms properly into account. However, there are two additional terms, which are responsible for the propensity of the method to overcompensate the BSSE. The magnitude of these minor terms depends on the size and quality of the basis and, in accordance with the accumulated numerical experience, ${ }^{26-28}$ decreases as the basis set is enlarged. It has generally been accepted that one almost always has to correct for the BSSE, and that adequate basis sets have to be used to avoid the overcompensating nature of the $\mathrm{CP}$ method.

This status quo seems to have been challenged by new results obtained from calculations using the aug-cc-pVXZ basis sets of Dunning. As most of the pioneering investigations $s^{5,13-15}$ have shown, it is rather important to correct for the BSSE if the standard cc-pVXZ basis sets are used for the calculations. In these cases, the $\mathrm{CP}$-corrected properties are nearly always closer to the available experimental data than the uncorrected ones. However, the situation is substantially different in the case of the aug-cc-pVXZ basis sets. The general trend is that the uncorrected aug-cc-pVXZ results lie close to the corresponding experimental values, and the convergence of the $\mathrm{CP}$-corrected aug-cc-pVXZ results to the CBS limit is slow. Based on these findings many of the authors of the pioneering studies ${ }^{14,15}$ criticized the CP method and questioned the applicability of the $\mathrm{CP}$ scheme in conjunction with the aug-cc-pVXZ basis sets.

Dunning and coworkers have recently published a review ${ }^{4}$ about their activity devoted to the exploration of the limits of the $\mathrm{CP}$ scheme. These authors have shown that, in many cases, the convergence behavior of various molecular properties is significantly improved if the calculations are corrected for the BSSE. According to Dunning and coworkers, ${ }^{4}$ for many investigated properties the smooth convergence behavior of the results obtained with augcc-pVXZ basis sets is a pure illusion, as it is due to a fortuitous cancellation of the BSSE and the basis set incompleteness error (BSIE). Correction for the BSSE destroys the balance of the two errors; consequently, the $\mathrm{CP}$-corrected data lie farther away from experiment than the uncorrected ones. However, the use of CP-corrected data is much safer for CBS limit extrapolation: the corrected data suffer only from the BSIE, which can be taken into account by a suitable extrapolation to the CBS limit. Anomalies related to the extrapolation to the CBS limit were most pronounced for weakly bound intermolecular complexes $^{4}$ (van der Waals and hydrogen-bonded systems), although similar behaviour was observed even for certain strongly bound systems. ${ }^{4}$

Dunning and coworkers have investigated various molecular properties like interaction energies and equilibrium distances of di- and triatomic molecules. However, their work on real manydimensional potential energy hypersurfaces (PESs) was limited to $(\mathrm{HF})_{2}$, partly due to the lack of an efficient automated procedure for BSSE-free geometry optimizations. [The optimized geometries were computed from numerical (CP-corrected or uncorrected) gradients.] Those studies, which expressed scepticism ${ }^{14,15}$ about the usefulness of the $\mathrm{CP}$ scheme when applied in connection with the aug-cc-pVXZ basis sets, were devoted to the characterization of PESs of hydrogen-bonded systems like the HF dimer ${ }^{15}$ and the water dimer. ${ }^{14,15}$

To resolve this apparent controversy, we decided to carry out geometry optimizations on prototypical hydrogen-bonded systems. Our goal is to investigate the behavior of the $\mathrm{CP}$ method in conjunction with the aug-cc-pVXZ basis sets for equilibrium geometric parameters. Therefore, we optimized the geometry of $(\mathrm{HF})_{2}$ and $\left(\mathrm{H}_{2} \mathrm{O}\right)_{2}$ employing analytical gradients at both the uncorrected and BSSE-corrected MP2/aug-cc-pVXZ [X $=\mathrm{D}(2)$, $\mathrm{T}(3), \mathrm{Q}(4)$, for $(\mathrm{HF})_{2}$ also 5] levels of theory. The geometrical data obtained provide evidence in support of Dunning's opinion described above. From the comparison of our data with the literature results available on $\left(\mathrm{H}_{2} \mathrm{O}\right)_{2}$, we evaluate the reliability of various approximations used in geometry optimizations (e.g., freezing a subset of the parameters), and show the importance of carrying out full geometry optimizations when extrapolation of geometrical parameters to the CBS limit is desired. Finally, we present optimized geometries and equilibrium interaction energies of the HF$\mathrm{H}_{2} \mathrm{O}$ complex obtained on both uncorrected and BSSE-corrected MP2/aug-cc-pVXZ (X $=\mathrm{D}, \mathrm{T}, \mathrm{Q})$ PESs. 


\section{Computational Details}

All $a b$ initio energy and gradient calculations utilized the Gaussian94 program system. ${ }^{29}$ For the $\mathrm{CP}$ geometry optimizations we used a program system described in ref. 30 with some small modifications in both the scripts and the various Gaussian links. The main idea behind the $\mathrm{CP}$ optimization is that various derivatives of eq. (6) can easily be calculated. For example, the gradient on the $\mathrm{CP}$-corrected surface can be calculated as

$$
\begin{aligned}
\frac{\delta E^{B B}}{\delta x}=\frac{\delta E_{A B}(A B)}{\delta x}+\frac{\delta E_{A}(A)}{\delta x} & -\frac{\delta E_{A}(A B)}{\delta x} \\
& +\frac{\delta E_{B}(B)}{\delta x}-\frac{\delta E_{B}(A B)}{\delta x},
\end{aligned}
$$

where $x$ is a geometry parameter. Our present implementation enables us to determine optimized geometries on the corrected PES in three to four optimization steps provided the initial geometry is the corresponding uncorrected optimized one. For rapid convergence it was important to use reliable Hessians in conjunction with simple Z-matrix-type coordinates during the GDIIS ${ }^{31}$ optimization. The Hessian was usually calculated on the uncorrected PES. In all correlated-level calculations the 1 s core orbitals of $\mathrm{O}$ and $\mathrm{F}$ have been kept frozen.

\section{Results and Discussion}

\section{HF DIMER}

The HF dimer (see Fig. 1) is one of the most important prototypes of hydrogen-bonded systems. Therefore, it has been the subject of detailed experimental (cf., ref. 32 and references therein) and theoretical (cf., ref. 13 and references therein) studies. The structure of $(\mathrm{HF})_{2}$ was studied at the MP2/augcc-pVXZ $(X=\mathrm{D}, \mathrm{T}$, and $\mathrm{Q})$ levels by Peterson and Dunning (henceforth, PD) ${ }^{13}$ In that article the geometry of the complex was fully optimized at the MP2/aug-cc-pVDZ and MP2/aug-cc-pVTZ levels on both the uncorrected and CP-corrected potential energy hypersurfaces. (PD used numerical gradients for the optimization.) With the aug-cc-pVQZ basis set PD carried out full geometry optimization on the uncorrected PES, while they optimized only the intermolecular F-F distance $\left(r_{\mathrm{FF}}\right.$, Fig. 1$)$ on the CP-corrected PES, keeping the other parameters fixed at their estimated MP2/aug-cc-pVQZ values. (For further details, see ref. 13.) The most important conclusions drawn by PD are as follows: (a) the uncorrected MP2/aug-cc-pVXZ interaction energies
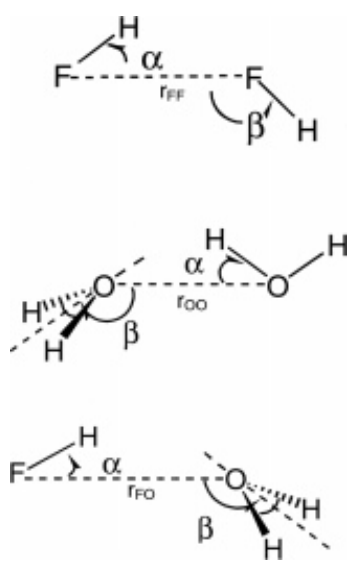

FIGURE 1. Geometrical parameters of the $(\mathrm{HF})_{2}$, $\left(\mathrm{H}_{2} \mathrm{O}\right)_{2}$, and $\mathrm{HF}-\mathrm{H}_{2} \mathrm{O}$ complexes.

and geometries are closer to experiment than the corresponding corrected ones; (b) convergence of the $\mathrm{CP}$-corrected binding energies and geometries is more regular than that of the uncorrected parameters; and (c) the CBS limit of the CP-corrected $r_{\mathrm{FF}}$ distance, $2.737 \AA$, is numerically indistinguishable from the uncorrected aug-cc-pVQZ optimized value. Overall, $P D$ stressed the importance of the $C P$ correction for the investigated properties.

As a first step in our investigation, we reoptimized the geometry of $(\mathrm{HF})_{2}$ at the levels for which PD published data. We were able to reproduce their results with only slight deviations by using our automated CP optimization algorithm (see Table I for the results). For the $\mathrm{CP}$-corrected MP2/aug-ccpVQZ case PD carried out partial optimizations by fixing most of the internal parameters. Our full optimization at the same level confirmed the adequacy of the approximations applied by PD, the fully optimized parameters $\left(\alpha=6.7^{\circ}, \beta=111.7^{\circ}\right.$, $r_{\mathrm{FF}}=2.753 \AA$ ) are close to the corresponding values $\left(\alpha=6.4^{\circ}, \beta=111.15^{\circ}, r_{\mathrm{FF}}=2.753 \AA\right.$ ) of PD. As mentioned above, the uncorrected MP2/augcc-pVQZ $r_{\mathrm{FF}}$ distance seems to be converged, and agrees with the CBS limit, $2.737 \AA$, obtained on the $\mathrm{CP}$-corrected surface by PD. (One has to note here that the CBS limit of $r_{\mathrm{FF}}$ determined by our geometry data, $2.741 \AA$, differs slightly from the corresponding value of $\mathrm{PD}, 2.737 \AA$ ). At the same time, the actual value of the $r_{\mathrm{FF}}$ distance $(2.753 \AA)$ on the corrected PES is far from both the MP2/augcc-pVQZ and the corrected CBS limit values. This situation provides an opportunity to further investigate the importance of $\mathrm{CP}$ correction in estimating the CBS limit of geometrical parameters, like the $r_{\mathrm{FF}}$ distance in $(\mathrm{HF})_{2}$. It seems to be worth investigat- 
PAIZS ET AL.

TABLE I.

Geometrical Parameters (Angles/Deg and Lengths/Å) and Total Energies $\left(E_{\text {tot }} / E_{h}\right)$ of the HF Dimer Calculated at the Uncorrected and CP-Corrected MP2/aug-cc-pVXZ $(X=2,3,4,5)$ Levels of Theory.

\begin{tabular}{|c|c|c|c|c|}
\hline \multicolumn{5}{|c|}{ Uncorrected PES } \\
\hline$x$ & $r_{\mathrm{FF}}$ & $\alpha$ & $\beta$ & $E_{\text {tot }}$ \\
\hline D & $2.753^{a}$ & $6.5\left(6.6^{a}\right)$ & $110.2\left(110.1^{\mathrm{a}}\right)$ & $-200.519075^{\circ}$ \\
\hline $\mathrm{T}$ & $2.746^{\mathrm{a}}$ & $6.4^{\mathrm{a}}$ & $111.1^{\mathrm{a}}$ & $-200.689285^{a}$ \\
\hline Q & $2.736\left(2.737^{a}\right)$ & $6.4^{\mathrm{a}}$ & $111.5\left(111.6^{\mathrm{a}}\right)$ & $-200.746906^{\circ}$ \\
\hline 5 & 2.739 & 6.3 & 112.0 & -200.768417 \\
\hline \multicolumn{5}{|c|}{ Corrected PES } \\
\hline$N$ & $r_{\mathrm{FF}}$ & $\alpha$ & $\beta$ & $E_{\text {tot }}$ \\
\hline D & $2.812\left(2.813^{\mathrm{a}}\right)$ & $7.0^{\mathrm{a}}$ & $111.3^{\mathrm{a}}$ & $-200.51806 C$ \\
\hline $\mathrm{T}$ & $2.770\left(2.772^{a}\right)$ & $6.7^{\mathrm{a}}$ & $111.7\left(111.8^{\mathrm{a}}\right)$ & -200.688525 \\
\hline Q & $2.753^{a, b}$ & 6.7 & 111.7 & -200.746495 \\
\hline 5 & 2.749 & 6.7 & 111.7 & $-200.76815 \varepsilon$ \\
\hline
\end{tabular}

${ }^{a}$ Ref. 6.

${ }^{b}$ Partial optimization. For details, see ref. 6 . For the definition of the variables, see Figure 1. In those cases, when two values are presented for a variable, our optimized value does not agree with that of ref. 6 given in parentheses.

ing the geometry of $(\mathrm{HF})_{2}$ at the MP2/aug-cc-pV5Z level for the following reasons. If convergence of $r_{\mathrm{FF}}$ was really manifested at the MP2/aug-cc-pVQZ level, the MP2/aug-cc-pV5Z value would coincide with the MP2/aug-cc-pVQZ value. On the other hand, any other MP2/aug-cc-pV5Z $r_{\text {FF }}$ value would question the extrapolation to the CBS limit using the uncorrected data. As it turns out, both the uncorrected $\left(\alpha=6.3^{\circ}, \beta=112.0^{\circ}, r_{\mathrm{FF}}=2.739 \AA\right)$ and CP-corrected $\left(\alpha=6.7^{\circ}, \beta=111.7^{\circ}, r_{\mathrm{FF}}=2.749 \AA\right)$ geometric parameters lie close to the corresponding MP2/aug-cc-pVQZ values. It is noted, that the difference between the corrected and uncorrected $r_{\mathrm{FF}}$ values is substantial, $0.010 \AA$, at the MP2/augcc-pV5Z level of theory. The series of MP2/aug-cc$\mathrm{pVXZ}(X=\mathrm{D}, \mathrm{T}, \mathrm{Q}, 5) r_{\mathrm{FF}}$ data represent a minimum curve with the minimum between $X$ equal to $Q$ and 5 (see Fig. 2). On the other hand, the $\mathrm{CP}$-corrected $\mathrm{MP2} /$ aug-cc-pVXZ $(\mathrm{X}=\mathrm{D}, \mathrm{T}, \mathrm{Q}, 5) r_{\mathrm{FF}}$ distances follow a monotonic curve.

One could extrapolate to the CBS limit of the $r_{\mathrm{FF}}$ distance in three ways using the available data obtained on the corrected PES; for example, one could use the $\{2,3,4\},\{3,4,5\}$, and $\{2,3,4,5\}$ series for determining extrapolated geometric parameters according to eq. (1). Obviously, more reasonable values are expected for the latter choices. The CBS limit values of the $r_{\mathrm{FF}}$ distance of $(\mathrm{HF})_{2}$ for the above listed series are reasonably close to each other at 2.741, 2.748, and $2.745 \AA$, respec- tively. (For the two to five fitting we used our own $\mathrm{CP}$-corrected data, which slightly differ from those of PD.) One has to note that the MP2/augcc-pVDZ level, which represents the lowest level of theory employed, provides a much poorer approximation than that obtained at the MP2/augcc-pVTZ level. This effect can be seen, for example, in the huge BSSE content of the geometry. After correction for BSSE, the $r_{\mathrm{FF}}$ distance is lengthened by $0.059,0.024,0.017 \AA$, and $0.010 \AA$ at the MP2/aug-cc-pVDZ, MP2/aug-cc-pVTZ, MP2/augcc-pVQZ, and MP2/aug-cc-pV5Z levels of theory,

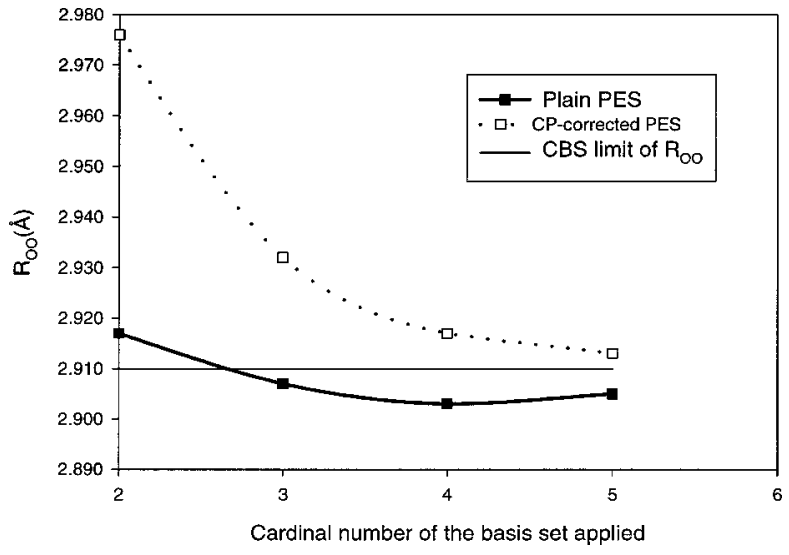

FIGURE 2. Comparison of the plain and CP-corrected $r_{\mathrm{FF}}$ distances for $(\mathrm{HF})_{2}$. 
respectively. Inferiority of the MP2/aug-cc-pVDZ geometry parameters, compared to the larger basis set results, is basically responsible for the differences between the CBS limit values obtained from the three fittings.

Nevertheless, as Chuang and Truhlar ${ }^{12}$ explored, one is tempted to use the inexpensive DZ and TZ data to obtain extrapolated geometric parameters corresponding to the basis set limit. However, instead of using the scheme of Chuang and Truhlar, we employed eq. (2) in the form of

$$
A_{\mathrm{X}}=A_{\mathrm{CBS}}+a \mathrm{X}^{-3}
$$

to obtain estimates of the extrapolated $r_{\mathrm{FF}}$. Our noteworthy result is that the extrapolated $r_{\mathrm{FF}}$ distances, 2.743 and $2.752 \AA$ in the uncorrected and BSSEcorrected cases, respectively, are very close to the corresponding MP2/aug-cc-pV5Z numbers. This is especially notable for the $\mathrm{CP}$-corrected $r_{\mathrm{FF}}$ distance, in which case the MP2/aug-cc-pVTZ value deviates from the MP2/aug-cc-pV5Z value by $0.021 \AA$, while the extrapolated distance deviates only by $0.003 \AA$.

In summary, the smooth convergence behavior of the geometric parameters obtained directly with the aug-cc-pVXZ basis sets is due to a fortuitous cancellation of BSSE and BSIE. The simple exponential or polynomial functions employed to extrapolate to the CBS limit cannot work reliably if the points do not follow a monotonic curve, as is the case for the uncorrected MP2/aug-cc-pVXZ results. On the other hand, although the $\mathrm{CP}$-corrected $r_{\mathrm{FF}}$ distances are usually farther away from the extrapolated values than the uncorrected distances (this is true for all but the MP2/aug-cc-pV5Z data), changes in the corrected geometric parameters are monotonic, and thus are in better accordance with the design philosophy of the aug-cc-pVXZ basis sets.

\section{WATER DIMER}

The water dimer (see Fig. 1) has a linear structure, which is known both from experiment ${ }^{33}$ and from $a b$ initio calculations [ref. 15, and references therein]. Our results and the available MP2/aug-ccpVXZ geometry data are summarized in Table II.

The first study devoted to the investigation of $\left(\mathrm{H}_{2} \mathrm{O}\right)_{2}$ employing the aug-cc-pVXZ $(\mathrm{X}=2,3,4,5)$ basis sets at the MP2 level was carried out by Feller, ${ }^{5}$ who determined the interaction energy of the complex at various correlated levels at a fixed geometry. The first geometry optimizations on the water dimer at the MP2(FC)/aug-cc-pVXZ $(X=D, T, Q)$ were carried out by Feller et al. ${ }^{34}$ on the uncorrected PES. Importance of fragment relaxation terms in the $\mathrm{CP}$

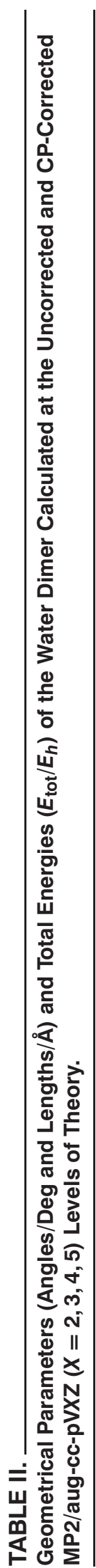


scheme was investigated by Xantheas, ${ }^{35}$ who determined the fully relaxed geometry of $\left(\mathrm{H}_{2} \mathrm{O}\right)_{2}$ at the MP2/aug-cc-pVDZ and MP2/aug-cc-pVTZ levels on the uncorrected potential energy hypersurfaces. Furthermore, Xantheas carried out partial optimizations at the MP2/aug-cc-pVQZ and MP2/aug-cc$\mathrm{pV} 5 \mathrm{Z}$ levels varying the intermolecular $r_{\mathrm{OO}}$ distance (Fig. 1) and keeping other parameters fixed at their MP2/aug-cc-pVTZ value. Xantheas' MP2/aug-ccpV5Z value for the $r_{\mathrm{OO}}$ distance is $2.905 \AA$ on the uncorrected and $2.913 \AA$ on the CP-corrected PESs, respectively. In a recent article, Halkier et al. ${ }^{15}$ also investigated the structure of the water dimer employing the MP2/aug-cc-pVXZ models. These authors performed constrained optimizations, for example, they kept the water monomer parameters frozen at the corresponding experimental values. With this approximation, the geometry of $\left(\mathrm{H}_{2} \mathrm{O}\right)_{2}$ was determined at the MP2/aug-cc-pVXZ $(X=2,3,4)$ levels at both the uncorrected and the $\mathrm{CP}$-corrected surfaces. (As a further approximation, the intermolecular valence angles were also frozen in the optimizations on the $\mathrm{CP}$-corrected PES at their corresponding uncorrected values.) One should note here that, based on the analysis of their data, Halkier and coworkers argued against the computation of $\mathrm{CP}$-corrected geometries at the MP2/aug-cc-pVXZ levels.

The results of our full geometry optimizations carried out on the uncorrected and $\mathrm{CP}$-corrected PESs are shown in Table II. At the MP2/aug-ccpVDZ and MP2/aug-cc-pVTZ levels we could reproduce Feller's and Xantheas' results with slight differences on both the uncorrected and corrected PESs. On the other hand, the $r_{\mathrm{OO}}$ distances obtained by Halkier et al. for the uncorrected surfaces differ considerably from the corresponding fully relaxed values. For example, the difference between the $r_{\mathrm{OO}}$ distances of Feller and Halkier is $0.016 \AA$ at the MP2/aug-cc-pVTZ level. This discrepancy questions the validity of the approximations utilized by Halkier et al. Furthermore, Xantheas' data determined at the MP2/aug-cc-pVQZ level from partial optimizations agree reasonably well with both Feller's and our values obtained from full optimizations. Hereby, we can confirm the adequacy of the approximations employed by Xantheas in his partial optimizations on $\left(\mathrm{H}_{2} \mathrm{O}\right)_{2}$, for example, keeping all the intramolecular and the valence bending intermolecular parameters at their optimized MP2/ aug-cc-pVTZ values in the subsequent aug-ccpVQZ and aug-cc-pV5Z optimizations. Because of the reliability of the approximations employed by Xantheas and the cost of the MP2/aug-cc-pV5Z op- timization we decided not to determine the geometry of $\left(\mathrm{H}_{2} \mathrm{O}\right)_{2}$ at the MP2/aug-cc-pV5Z level.

Concerning the extrapolation of the $r_{\mathrm{OO}}$ distance to the CBS limit, one can draw the following conclusions. The two series of $r_{\mathrm{OO}}$ distances, determined by Feller et al., Xantheas and Halkier et al. employing different approximations during the optimizations, follow a curve with a minimum. That is, Halkier's aug-cc-pVTZ $r_{\mathrm{OO}}$ distance at $2.891 \AA$ is shorter than the corresponding aug-cc-pVDZ $(2.912 \AA)$ and aug-cc-pVQZ $(2.895 \AA)$ values. This behavior is presumably due to the inadequacy of the constraints employed by Halkier et al. during the optimizations. In the data series of Feller et al. and Xantheas, the aug-cc-pVQZ $r_{\mathrm{OO}}$ distance $(2.903 \AA)$ is shorter than the corresponding augcc-pVDZ (2.916 $\AA)$, aug-cc-pVTZ $(2.907 \AA)$, and aug-cc-pV5Z (2.905 $\AA$ ) values. This behavior of the aug-cc-pVXZ basis sets in the case of uncorrected calculations is very similar to what we observed in full geometry optimizations on $(\mathrm{HF})_{2}$. On the other hand, the $\mathrm{CP}$-corrected $r_{\mathrm{OO}}$ distances converge more regularly, all three data series obtained by Xantheas, Halkier et al. and the present work ascend gradually when enlarging the cardinal number of the employed basis set (see Fig. 3). The extrapolated CBS limit of the $r_{\mathrm{OO}}$ distance obtained from Halkier's data seems to be too short at $2.897 \AA$, indicating again the inadequacy of the constraints employed during the geometry optimizations. On the other hand, the extrapolated $r_{\mathrm{OO}} \mathrm{CBS}$ limit values obtained from Xantheas' partially optimized aug-cc-pVXZ $\{X=2,3,4,5\}$ and our fully optimized aug-cc-pVXZ $\{X=2,3,4\}$ data using eq. (1) practically coincide at $2.910 \AA$. It is worth noting in this respect that the CBS limit values determined from

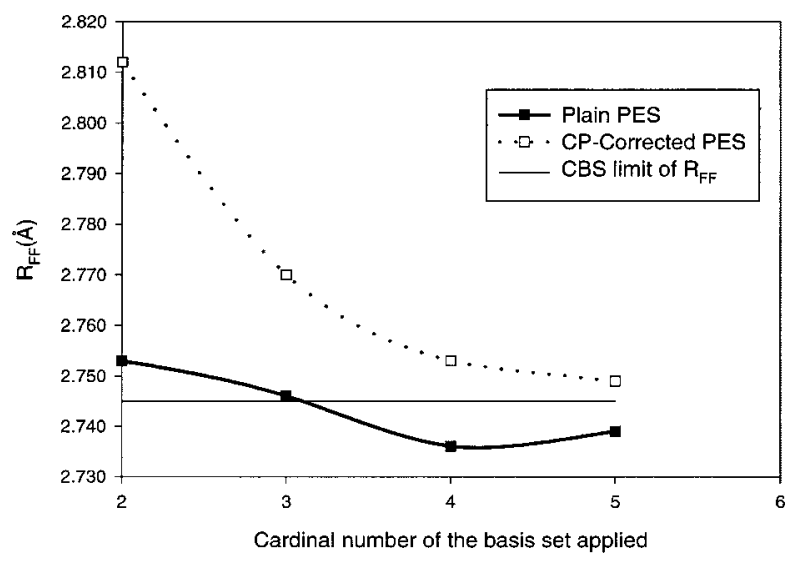

FIGURE 3. Comparison of the plain and CP-corrected $r_{\mathrm{OO}}$ distances for $\left(\mathrm{H}_{2} \mathrm{O}\right)_{2}$. 
the $\{X=2,3,4\}$ and $\{X=3,4,5\}$ series of Xantheas are also the same at $2.910 \AA$.

Similarly to the case of $(\mathrm{HF})_{2}$, use of eq. (8) for the extrapolation of aug-cc-pVDZ and aug-ccpVTZ data gives very satisfactory results for the $\mathrm{CP}$-corrected $r_{\mathrm{OO}}$ distances. While the difference between the aug-cc-pVTZ and aug-cc-pV5Z $r_{\mathrm{OO}}$ distances is $0.020 \AA$ the extrapolated value differs by only $0.005 \AA$.

As a summary of the above considerations, we strongly advocate to perform full geometry optimizations in those studies whose aim is the extrapolation of geometrical parameters to the CBS limit. The reliability of the CBS limit of the $r_{\mathrm{OO}}$ distance determined from the fully relaxed $\mathrm{CP}$-corrected aug-cc-pVXZ $\{X=2,3,4\}$ potential energy hypersurfaces is appealing compared to the aug-cc-pVXZ $\{X=2,3,4,5\}$ data obtained by Xantheas. ${ }^{35}$

\section{HF- $\mathrm{H}_{2} \mathrm{O}$ COMPLEX}

Determination of the structure of the $\mathrm{HF}-\mathrm{H}_{2} \mathrm{O}$ complex proved to be a challenge for experimentalists and theoreticians alike. Theoretical studies ${ }^{36,37}$ showed that one can find stationary points on the $\mathrm{HF}-\mathrm{H}_{2} \mathrm{O}$ PES with both $\mathrm{C}_{\mathrm{s}}$ and $\mathrm{C}_{2 \mathrm{v}}$ symmetries. (In the case of the $C_{2 v}$ structure the $\alpha$ and $\beta$ angles (Fig. 1) are 0 and 180 degrees, respectively.) The $C_{2 v}$ species is favored by certain electrostatic arguments. ${ }^{38}$ Namely, the $\mathrm{C}_{2 \mathrm{v}}$ arrangement of the complex allows the dipole moment of $\mathrm{H}_{2} \mathrm{O}$ to be aligned with the dipole of HF. On the other hand, the interaction of the negative charges of the respective quadrupole tensor element of $\mathrm{H}_{2} \mathrm{O}$ and the positive end of the HF dipole clearly favors a structure where the plane of $\mathrm{H}_{2} \mathrm{O}$ is perpendicular to the direction of the $\mathrm{HF}$ dipole. The structure of $\mathrm{HF}-\mathrm{H}_{2} \mathrm{O}$ can be considered as a compromise between these electrostatic and other (less important) nonelectrostatic effects. Indeed, although the $C_{s}$ structure is more stable than the $C_{2 v}$ one, the energy difference between them is only 0.1 and $0.5 \mathrm{kcal} \mathrm{mol}^{-1}$ using a small basis set at the SCF and MP2 levels, ${ }^{36}$ respectively. The experimental ${ }^{39}$ estimate of the barrier is $0.4 \mathrm{kcal} \mathrm{mol}^{-1}$. Experimental determination of the angular features of the $\mathrm{HF}-\mathrm{H}_{2} \mathrm{O}$ complex is difficult. ${ }^{30}$ The energy barrier between the $\mathrm{C}_{\mathrm{s}}$ and $C_{2 v}$ structures is small, presumably close to the energy of the first vibrational level. In the case when the first vibrational level occurs above the top of the barrier, the experiment would predict a planar structure despite the existence of a double-well potential. If the vibrational level is slightly below the top of the barrier, the experiment can predict only a poorly defined structure.

The most recent theoretical investigation devoted to the structure of $\mathrm{HF}-\mathrm{H}_{2} \mathrm{O}$, including geometry optimizations, was carried out by Novoa et al. ${ }^{37}$ at the MP2 /6-311++G(2d,2p) level of theory resulting in an energy difference of $0.45 \mathrm{kcal} \mathrm{mol}^{-1}$ between the $C_{s}$ and $C_{2 v}$ structures. They have also calculated the equilibrium $r_{\mathrm{FO}}$ distance $(2.663 \AA)$ using a constrained optimization keeping the monomer parameters fixed. Finally, their interaction energies computed at the uncorrected and $\mathrm{CP}$-corrected MP2/6-311++G(2d,2p) levels are -9.20 and -7.87 $\mathrm{kcal} \mathrm{mol}^{-1}$, respectively.

Because of the available experimental and theoretical information, the $\mathrm{HF}-\mathrm{H}_{2} \mathrm{O}$ complex is an ideal subject to test the CBS limit values of various properties determined from calculations performed at the MP2/aug-cc-pVXZ $\{X=2,3,4\}$ levels. To explore the quality of theoretical results obtained on the uncorrected and $\mathrm{CP}$-corrected surfaces, we decided to calculate the equilibrium dissociation energy, the energy difference between the $C_{s}$ and $\mathrm{C}_{2 \mathrm{v}}$ species, and the equilibrium geometry of HF$\mathrm{H}_{2} \mathrm{O}$. The experimental data were determined by Legon and coworkers ${ }^{39-41}$ using microwave rotational spectroscopy. The calculated total energies, energy barriers, and geometrical parameters obtained at the MP2/aug-cc-pVXZ $\{X=2,3,4\}$ levels are given in Table III. The CBS value for the total energy of $\mathrm{H}_{2} \mathrm{O}$ is $-76.363558 E_{h}$, obtained from the total energies $(-76.260910,-76.328992$, and $-76.351919 E_{h}$ ) calculated at the MP2/aug-ccpVDZ, MP2/aug-cc-pVTZ, and MP2/aug-cc-pVQZ levels, respectively.

The experimental ${ }^{41}$ equilibrium interaction energy ( $E_{\text {int }}$ ) of $\mathrm{HF}-\mathrm{H}_{2} \mathrm{O}$ is $-10.2 \mathrm{kcal} \mathrm{mol}^{-1}$, determined by Legon and coworkers from absolute intensities of rotational transitions. As mentioned before, the best theoretical values ${ }^{37}$ available are -9.20 and $-7.87 \mathrm{kcal} \mathrm{mol}^{-1}$ obtained from uncorrected and CP-corrected calculations using a medium-size basis set and the MP2 method. The uncorrected aug-cc-pVXZ $\{X=2,3,4\}$ interaction energies shown in Table IV are appealingly close to each other. The CBS limit value of $E_{\text {int }}$ obtained using the extrapolated total energies of the monomers and the complex, differs only slightly from these values. The interaction energies obtained at the CP-corrected PESs considerably differ from the corresponding uncorrected energies. However, this difference gradually decreases, considering the series of basis sets, going from the aug-cc-pVDZ set to the aug-cc-pVQZ one. The CP-corrected CBS 
TABLE III.

Geometrical Parameters (Angles/Deg and Lengths/Å) and Total Energies $\left(E_{\text {tot }} / E_{h}\right)$ of $\mathrm{HF}-\mathrm{H}_{2} \mathrm{O}$ Calculated at the Uncorrected and CP-Corrected MP2/aug-cc-pVXZ $(X=2,3,4)$ Levels of Theory.

\begin{tabular}{|c|c|c|c|c|c|c|c|c|}
\hline \multirow[b]{2}{*}{$x$} & \multicolumn{4}{|c|}{ Plain } & \multicolumn{4}{|c|}{ CP-Corrected } \\
\hline & $E_{\text {tot }}$ & $r_{\mathrm{FO}}$ & $\alpha$ & $\beta$ & $E_{\text {tot }}$ & $r_{\mathrm{FO}}$ & $\alpha$ & $\beta$ \\
\hline \multicolumn{9}{|c|}{$\mathrm{C}_{\mathrm{s}}$} \\
\hline 2 & -176.531107 & 2.658 & 129.5 & 1.4 & -176.529305 & 2.701 & 130.6 & 1.5 \\
\hline 3 & -176.684156 & 2.643 & 130.7 & 1.4 & -176.683198 & 2.662 & 132.0 & 1.5 \\
\hline 4 & -176.735847 & 2.640 & 132.4 & 1.4 & -176.735257 & 2.654 & 132.6 & 1.4 \\
\hline CBS & -176.762208 & 2.639 & - & - & -176.761869 & 2.652 & - & - \\
\hline \multicolumn{9}{|c|}{$\mathrm{C}_{2 \mathrm{v}}$} \\
\hline 2 & -176.530314 & 2.666 & 0.0 & 180.0 & -176.528674 & 2.710 & 0.0 & 180.0 \\
\hline 3 & -176.683503 & 2.655 & 0.0 & 180.0 & -176.682605 & 2.670 & 0.0 & 180.0 \\
\hline 4 & -176.735318 & 2.648 & 0.0 & 180.0 & -176.734751 & 2.661 & 0.0 & 180.0 \\
\hline CBS & -176.761803 & 2.636 & - & - & -176.761465 & 2.658 & - & - \\
\hline
\end{tabular}

limit of $E_{\text {int }}$ at $-8.6 \mathrm{kcal} \mathrm{mol}^{-1}$ is remarkably close to the corresponding uncorrected value at $-8.8 \mathrm{kcal}$ $\mathrm{mol}^{-1}$. Comparing the experimental and theoretical results, one has to note that the CBS limit $\Delta E$ values obtained at the MP2 level considerably differ from the experimental ${ }^{40}$ value $(-10.2 \mathrm{kcal} / \mathrm{mol})$. Perhaps, truncation of the correlation energy expansion at the MP2 level does not represent a satisfactory description of the investigated problem.

The calculated energy barriers $(\Delta E)$ belonging to the transition from the $C_{s}$ to the $C_{2 v}$ species of HF$\mathrm{H}_{2} \mathrm{O}$ are shown in Table IV. $\Delta E$ gradually decreases, going from the smallest to the larger basis sets. It is worth noting that the $\Delta E$ values obtained at the plain and $\mathrm{CP}$-corrected surfaces are very close to each other at the MP2/aug-cc-pVQZ level of theory. The CBS limit values of the same parameter obtained at the plain and the CP-corrected PESs numerically coincide at $0.25 \mathrm{kcal} \mathrm{mol}^{-1}$. The CBS limit of $\Delta E$ is considerably smaller than the corresponding equilibrium experimenta ${ }^{40}$ data $(0.36 \mathrm{kcal}$ $\mathrm{mol}^{-1}$ ), again showing the importance of inclusion of higher order correlation terms. It is worth noting that the good agreement with experiment obtained in previous theoretical studies ${ }^{36,37}$ is clearly due to cancellation of errors, for example, the quality of the basis sets employed in those investigations was unsatisfactory to consistently deal with the problems of BSSE and BSIE.

Analyzing the geometrical data (mainly the parameter $r_{\mathrm{FO}}$ ) presented in Table III, one can find trends similar to those already detected in the cases of $(\mathrm{HF})_{2}$ and $\left(\mathrm{H}_{2} \mathrm{O}\right)_{2}$. The $r_{\mathrm{FO}}$ values obtained on the uncorrected PES change less than the corresponding $\mathrm{CP}$-corrected values with change in the basis set. The CBS limit of $r_{\mathrm{FO}}$ at the uncorrected PES, $2.639 \AA$, is very close to both the MP2/aug-cc-pVTZ $(2.643 \AA)$ and MP2/aug-cc-pVQZ (2.640 $)$ ) values.

TABLE IV.

Equilibrium Interaction Energies $\left(E_{\text {tot }}\right.$ in $\left.\mathrm{kcal} / \mathrm{mol}\right)$ and Energy Splitting $\left(\Delta E\right.$ in kcal mol $\left.{ }^{-1}\right)$ between the $C_{s}$ and $\mathrm{C}_{2 \mathrm{v}}$ Species of $\mathrm{HF}-\mathrm{H}_{2} \mathrm{O}$ Calculated at the Uncorrected and CP-Corrected MP2/aug-cc-pVXZ $(X=2,3,4)$ Levels of Theory.

\begin{tabular}{|c|c|c|c|c|}
\hline \multirow[b]{2}{*}{$x$} & \multicolumn{2}{|c|}{$E_{\text {int }}$} & \multicolumn{2}{|c|}{$\Delta E$} \\
\hline & Plain & CP-Corrected & Plain & CP-Corrected \\
\hline 2 & -9.0 & -7.9 & 0.50 & 0.40 \\
\hline 3 & -9.0 & -8.4 & 0.41 & 0.37 \\
\hline 4 & -8.9 & -8.5 & 0.33 & 0.32 \\
\hline $\mathrm{CBS}^{\mathrm{a}}$ & -8.8 & -8.6 & 0.25 & 0.25 \\
\hline
\end{tabular}

a The CBS limit values were calculated using the respective CBS limit total energies shown in Table III. 


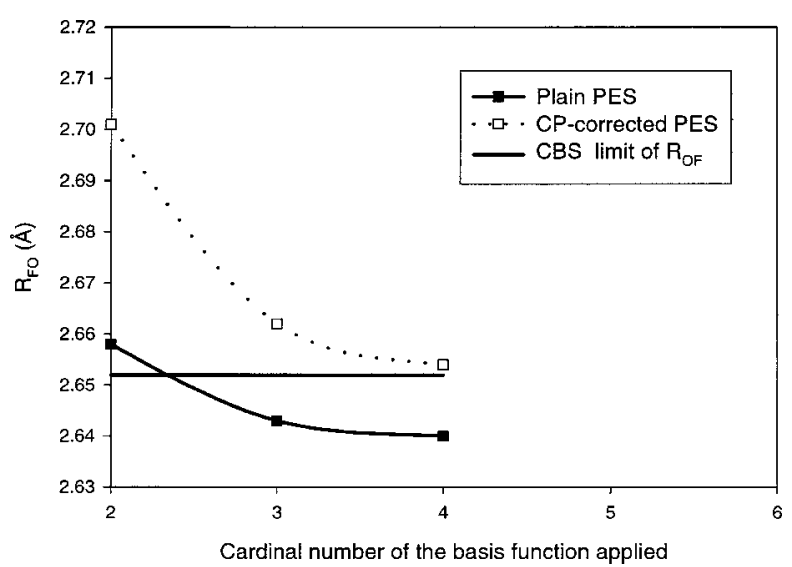

FIGURE 4. Comparison of the plain and CP-corrected $r_{\mathrm{FO}}$ distances for $\mathrm{HF}-\mathrm{H}_{2} \mathrm{O}$.

On the other hand, the CBS limit of $r_{\mathrm{FO}}$ is $2.652 \AA$ on the $\mathrm{CP}$-corrected PES, representing a difference of $0.013 \AA$ between the two extrapolated values. Legon and coworkers have determined the experimental value of $r_{\mathrm{FO}}$ at 2.662 å from the microwave rotational spectrum ${ }^{40}$ of $\mathrm{HF}-\mathrm{H}_{2} \mathrm{O}$. Direct comparison of the experimental $r_{0}$ and theoretical $r_{e}$ values is not fully valid, but it is clear that the $\mathrm{CP}$-corrected CBS limit $r_{\mathrm{FO}}$ distance lies closer to experiment than the corresponding uncorrected value (see Fig. 4). However, one has to note here that the vibrational effects on hydrogen bond lengths can be substantial resulting in a case when the uncorrected CBS limit value is closer to the unmeasured experimental $r_{e}$ value. Another interesting anomaly concerns the change of the actual value of $r_{F O}$ going from the $C_{s}$ to the $C_{2 v}$ species. One expects that the intermolecular bond length increases when climbing the transititon state $\left(C_{2 v}\right.$ species) region. All the data listed in Table III supports this statement, but the CBS limit of $r_{\mathrm{FO}}$ obtained at the plain PESs contradicts it. In our opinion, this strange behavior is again due to the unreliability of the CBS limit values of geometry parameters obtained on the uncorrected PES.

Concerning the extrapolated $r_{\mathrm{FO}}$ distances obtained by using eq. (8), one finds tendencies similar to those observed in the cases of $(\mathrm{HF})_{2}$ and $\left(\mathrm{H}_{2} \mathrm{O}\right)_{2}$. The extrapolated $\mathrm{C}_{2 \mathrm{v}} r_{\mathrm{FO}}$ distances, 2.650 and $2.653 \AA$, in the uncorrected and CP-corrected cases, respectively, are very close to the corresponding CBS value (2.658 $\AA$ ) determined by using eq. (1) on the $\mathrm{CP}$-corrected PESs. The extrapolated $\mathrm{C}_{\mathrm{S}} r_{\mathrm{FO}}$ distances, 2.637 and $2.646 \AA$ determined on the plain and CP-corrected PESs lie close to the corresponding CBS limit values (2.639 and 2.652 å) obtained by using eq. (1). It is worth noting here that the extrapolated [eq. (8)] $C_{2 v} r_{F O}$ value is longer than the corresponding $C_{s}$ value in both the plain and $\mathrm{CP}$-corrected cases. This means that bond length extrapolation based on eq. (8) is more reliable in the case of the $\mathrm{HF}-\mathrm{H}_{2} \mathrm{O}$ complex than application of eq. (1) because the expected change of parameter $r_{\mathrm{FO}}$ is at least qualitatively given back by the former technique.

\section{COMMENT ON THE TRENDS OF INTERMOLECULAR DISTANCES IN THE (HF) $)_{2}$, $\left(\mathrm{H}_{2} \mathrm{O}\right)_{2}$, AND HF- $\mathrm{H}_{2} \mathrm{O}$ SERIES}

One of the referees of the present article suggested to investigate trends observed for the intermolecular bond lengths in the $(\mathrm{HF})_{2},\left(\mathrm{H}_{2} \mathrm{O}\right)_{2}$, and $\mathrm{HF}-\mathrm{H}_{2} \mathrm{O}$ series. The two most interesting observations are as follows: (1) the differences between the plain aug-cc-pVQZ and the corresponding (CPcorrected) CBS values of the intermolecular distances are $0.005-0.008,0.007$, and $0.012 \AA$ for $(\mathrm{HF})_{2}$, $\left(\mathrm{H}_{2} \mathrm{O}\right)_{2}$, and $\mathrm{HF}-\mathrm{H}_{2} \mathrm{O}$, respectively; (2) the differences between the $\mathrm{CP}$-corrected aug-cc-pVQZ and (CP-corrected) CBS values of the intermolecular bond lengths are 0.008-0.012, 0.007, and $0.002 \AA$, gradually decreasing in the $(\mathrm{HF})_{2},\left(\mathrm{H}_{2} \mathrm{O}\right)_{2}$, and HF$\mathrm{H}_{2} \mathrm{O}$ series. (It is to be noted that three different CBS values were obtained for $(\mathrm{HF})_{2}$, resulting in uncertainties in the above-mentioned differences between the aug-cc-pVQZ and CBS limit values.)

We begin with the explanation of tendency (2), because in this case differences between two BSSEfree quantities are evaluated resulting in a less complex situation than that of point (1). The interaction between the monomers of the complexes in the $(\mathrm{HF})_{2},\left(\mathrm{H}_{2} \mathrm{O}\right)_{2}$, and $\mathrm{HF}-\mathrm{H}_{2} \mathrm{O}$ series is getting definitely stronger. This means that the importance of very weak interactions, like dispersion forces, is decreasing, while the strength of the electrostatic and charge transfer interactions is increasing for the series of complexes investigated. Of course, one expects more reliable results for $\mathrm{HF}-\mathrm{H}_{2} \mathrm{O}$ than for $(\mathrm{HF})_{2}$ by using the same basis set in the calculations. Equivalent with this statement is that the real physical part of the interaction is more satisfied (e.g., it is getting closer to the CBS limit) for $\mathrm{HF}-\mathrm{H}_{2} \mathrm{O}$ than for $(\mathrm{HF})_{2}$ at a given level of theory provided that a reasonable basis set is applied in the particular calculations.

The difference between the plain aug-cc-pVQZ and (CP-corrected) CBS limit bond distances depends clearly on the magnitude of the BSSE and BSIE. The magnitude of the BSSE also depends on 
two main factors in our case. The first and more important is the choice of the basis set applied. Plotting the BSSE content of the interaction energy vs. different basis sets results in a curve that exhibits a maximum. ${ }^{42}$ The flexibility of a small basis set is insufficient for the appearance of a significant BSSE-type correlation energy contribution. With the growing size of the basis set such contributions appear and the BSSE content of the interaction energy increases. Using even larger basis sets the BSSE content will again decrease. It is clear that the basis sets applied in the present study represent the postmaximum regions of such BSSE content curves, but the actual locations of the aug-cc-pVXZ $(X=$ $2,3,4$, and 5) points and the slopes of the curves are clearly different for the $(\mathrm{HF})_{2},\left(\mathrm{H}_{2} \mathrm{O}\right)_{2}$, and $\mathrm{HF}-\mathrm{H}_{2} \mathrm{O}$ cases. In this respect, similarly to the description of the physical part of the interaction, the strength of the interaction is very important. One is evidently closer to the saturated description at the MP2/augcc-pVQZ level of theory in the case of $\mathrm{HF}-\mathrm{H}_{2} \mathrm{O}$ than for $(\mathrm{HF})_{2}$. However, among other factors, the magnitude of the BSSE depends also on the intermolecular distance between the monomers. The shorter distance means more possibilities for BSSE-type delocalizations. The data presented in Tables I-III show that the shortest intermolecular distance is obtained for $\mathrm{HF}-\mathrm{H}_{2} \mathrm{O}$, where the intermolecular bond length is $0.25 \AA$ shorter than the corresponding value obtained for $\left(\mathrm{H}_{2} \mathrm{O}\right)_{2}$. The intermolecular distance for $(\mathrm{HF})_{2}$ is between the corresponding $\mathrm{HF}-\mathrm{H}_{2} \mathrm{O}$ and $\left(\mathrm{H}_{2} \mathrm{O}\right)_{2}$ values. The actual magnitude of the BSSE is determined by the interplay of these and other (less important) effects highly depending on the structure, interaction, basis set, etc. For the systems investigated in the present study, this interplay results that the BSSE contents of the intermolecular distances are $0.017,0.014$, and $0.014 \AA$ at the MP2/aug-ccpVQZ level for $(\mathrm{HF})_{2},\left(\mathrm{H}_{2} \mathrm{O}\right)_{2}$, and HF$\mathrm{H}_{2} \mathrm{O}$, respectively, showing approximately the same magnitude for these systems.

We found a reasonable explanation for the decreasing differences between the $\mathrm{CP}$-corrected intermolecular distances and their CBS limit for the systems investigated. We also saw that the actual BSSE content seems to be more or less constant for the $(\mathrm{HF})_{2},\left(\mathrm{H}_{2} \mathrm{O}\right)_{2}$, and $\mathrm{HF}-\mathrm{H}_{2} \mathrm{O}$ complexes at the MP2/aug-cc-pVQZ level of theory. Because the plain and CP-corrected MP2/aug-cc-pVQZ intermolecular distances bracket the CBS limit of the parameter investigated, the tendency that the difference between the plain aug-cc-pVQZ results and the corresponding CBS limit is increasing in the $(\mathrm{HF})_{2}$, $\left(\mathrm{H}_{2} \mathrm{O}\right)_{2}$, and $\mathrm{HF}-\mathrm{H}_{2} \mathrm{O}$ series, is explained. It is im- portant to note here that these observations further stress the importance of correction for the BSSE.

\section{Conclusions}

Geometry optimizations were carried out for the $(\mathrm{HF})_{2},\left(\mathrm{H}_{2} \mathrm{O}\right)_{2}$, and $\mathrm{HF}-\mathrm{H}_{2} \mathrm{O}$ intermolecular complexes using the MP2/aug-cc-pVXZ $\{X=2,3,4$, and 5$\}$ theoretical models on both the uncorrected and CP-corrected potential energy hypersurfaces. Our results and the available literature data clearly show that extrapolation of intermolecular distances to the CBS limit is satisfactory on PESs corrected for BSSE. Evaluation of two extrapolation schemes [eqs. (1) and (8)] suggests that reliable extrapolated geometry parameters can be obtained even with a simple two-point formula based on aug-cc-pVDZ and aug-cc-pVTZ data. Application of the extrapolation to obtain the CBS limit of intermolecular geometry parameters seems to be worth it if various species are to be compared on the PES of the complex investigated. Also, fixing intramolecular parameters at their experimental values could cause difficulties during the extrapolation. As the available literature data and our results clearly show, the MP2/aug-cc-pVXZ $\{X=2,3,4\}$ data series of intermolecular distances obtained from the $\mathrm{CP}$-corrected surfaces can be safely used for the purpose of CBS extrapolations.

\section{Acknowledgments}

P.S. expresses his gratitude to the Commission of the European Union for a scholarship that made his visit to Heidelberg possible.

\section{References}

1. Dunning, T. H., Jr. J Chem Phys 1989, 90, 1007.

2. Kendall, R. A.; Dunning, T. H., Jr.; Harrison, R. J. J Chem Phys 1992, 96, 6796.

3. Woon, D. E.; Dunning, T. H., Jr. J Chem Phys 1993, 98, 1358.

4. van Mourik, T.; Wilson, A. K.; Peterson, K. A.; Woon, D. E.; Dunning, T. H., Jr. Adv Quant Chem 1998, 31, 105, and references therein.

5. Feller, D. J Chem Phys 1992, 96, 6104.

6. Császár, A. G.; Allen, W. D.; Schaefer, H. F., III. J Chem Phys 1998, 108, 9751.

7. Martin, J. M. L. Chem Phys Lett 1996, 259, 669.

8. Feller, D. J Chem Phys 1993, 98, 7059.

9. Klopper, W.; Bak, K. L.; Jørgensen, P.; Olsen, J.; Helgaker, T. J Phys B 1999, 32, R103. 
10. Tarczay, Gy.; Császár, A. G.; Klopper, W.; Szalay, V.; Allen, W. D.; Schaefer, H. F., III. J Chem Phys 1999, 110, 11971.

11. Helgaker, T.; Klopper, W.; Koch, H. Noga, J. J Chem Phys 1997, 106, 9639.

12. Chuang, Y.; Truhlar, D. G. J Phys Chem A 1999, 103, 651.

13. Peterson, K. A.; Dunning, T. H., Jr. J Chem Phys 1995, 102, 2032.

14. Feyereisen, M. W.; Feller, D.; Dixon, D. A. J Phys Chem 1996, 100, 2993.

15. Halkier, A.; Koch, H.; Jørgensen, P.; Christiansen, O.; Nielsen, I. M. B.; Helgaker, T. Theor Chem Acc 1997, 97, 150.

16. Jansen, H. B.; Ross, P. Chem Phys Lett 1969, 3, 140.

17. Boys, S. B.; Bernardi, F. Mol Phys 1970, 19, 553.

18. Mayer, I. Int J Quantum Chem 1983, 23, 341.

19. Mayer, I. In Modelling of Structure and Properties of Molecules; Maksic, Z. B., Ed.; Ellis Horwood: Chichester, UK, 1987, p. 145.

20. Mayer, I.; Valiron, P. J Chem Phys 1998, 109, 3360.

21. Mayer, I. Int J Quantum Chem 1998, 70, 41.

22. Mayer, I.; Surján, P. R. Chem Phys Lett 1992, 191, 497.

23. Valiron, P.; Mayer, I. Chem Phys Lett 1997, 275, 46.

24. Daudey, J. P.; Claverie, P.; Malrieu, J. P. Int J Quantum Chem 1974, 8, 1 .

25. Mayer, I.; Túri, L. J Mol Struct (Theochem) 1991, 227, 43.

26. Gutowski, M.; van Lenthe, J. H.; Verbeek, J.; van Duijneveldt, F. B. Chem Phys Lett 1986, 124, 370.

27. Gutowski, M.; van Duijneveldt-van de Rijdt, J. G. C. M.; van Lenthe, J. H.; van Duijneveldt, F. B. J Chem Phys 1993, 98,4728 .

28. van Duijneveldt, F. B.; van Duijneveldt-van de Rijdt, J. G. C. M.; van Lenthe, J. H. Chem Rev 1994, 94, 1873.
29. Frisch, M. J.; Trucks, G. W.; Schlegel, H. B.; Gill, P. M. W.; Johnson, B. G.; RoCP, M. A.; Cheeseman, J. R.; Keith, T. A.; Petersson, G. A.; Montgomery, J. A.; Raghavachari, K.; AlLaham, M. A.; Zakrzewski, V. G.; Ortiz, J. V.; Foresman, J. B.; Cioslowski, J.; Stefanov, B. B.; Nanayakkara, A.; Challacombe, M.; Peng, C. Y.; Ayala, P. Y.; Chen, W.; Wong, M. W.; Andres, J. L.; Replogle, E. S.; Gomperts, R.; Martin, R. L.; Fox, D. J.; Binkley, J. S.; Defrees, D. J.; Baker, J.; Stewart, J. P.; Head-Gordon, M.; Gonzalez, C.; Pople, J. A. Gaussian, Inc.: Pittsburgh, PA, 1995.

30. Simon, S.; Duran, M.; Dannenberg, J. J. J Chem Phys 1996, 105, 11024.

31. Császár, P.; Pulay, P. J Mol Struct 1984, 114, 31.

32. Bohac, E. J.; Marshall, M. D.; Miller, R. E. J Chem Phys 1992, 96, 6681.

33. Dyke, T. R.; Mack, K. M.; Muenter, J. S. J Chem Phys 1977, 66, 498.

34. Feller, D.; Glendening, E. D.; Kendall, R. A.; Peterson, K. A. J Chem Phys 1994, 100, 4981.

35. Xantheas, S. S. J Chem Phys 1996, 104, 8821.

36. Szczesniak, M. M.; Scheiner, S.; Bouteiller, Y. J Chem Phys 1984, 81, 5024, and references therein.

37. Novoa, J. J.; Planas, M.; Whangbo, M.; Williams, J. M. Chem Phys 1994, 186, 175, and references therein.

38. Scheiner, S. Hydrogen Bonding; Oxford University Press: New York, 1997.

39. Legon, A. C.; Millen, D. J. Faraday Discuss Chem Soc 1982, 73, 71 .

40. Bevan, J. W.; Kisiel, Z.; Legon, A. C.; Millen, D. J.; Rogers, S. C. Proc R Soc A 1980, 372, 441.

41. Legon, A. C.; Millen, D. J.; North, H. M. Chem Phys Lett 1987, 135, 303.

42. Paizs, B.; Suhai, S. J Comput Chem 1998, 19, 575. 